\title{
Analysis of Arbitrarily Shaped Dielectric Lens Antenna
}

\author{
Mitsuo TAGUCHI Mitsuhiro MASUDA* Hideaki SHIMODA** \\ Kazumasa TANAKA \\ Dept. of Electrical \& Electronic Eng., Nagasaki University \\ 1-14 Bunkyo-machi, Nagasaki-shi, 852-8521, Japan \\ ** Telecom Technology Development Center, TDK Co. \\ 2-15-7, Higashi-Ohwada, Ichikawa-shi, Chiba, 272-0026 Japan
}

\section{Introduction}

The collision warning radar installed on the vehicle has been watched attention as the next safety driving support system in Japan. An electronically scanned MMWantenna, a microstrip antenna and a dielectric lens antenna have been proposed for this application [1]. The lens antenna has suitable characteristics such as narrow beamwidth, low sidelobes, and scanning capability for the collision warning radar. In this paper, the ray tracing method is proposed to analyze an arbitrarily shaped dielectric lens antenna.

\section{Theoretical analysis}

Figure 1 shows the dielectric lens antenna and the coordinate system for the ray tracing method. Let the source point on the primary radiator be $P^{\prime}\left(x_{0}, y_{0}, z_{0}\right)$. The equivalent electric and magnetic currents $J_{y}\left(x_{0}, y_{0}\right) \boldsymbol{i}_{y}$ and $M_{x}\left(x_{0}, y_{0}\right) \boldsymbol{i}_{x}$ are assumed on the source point $P^{\prime} . \quad \boldsymbol{i}_{x}, \boldsymbol{i}_{y}$ and $\boldsymbol{i}_{z}$ are the unit vectors of the Cartesian coordinate system. The flat inner surface of lens is $S_{1}$ and the outer surface of lens is $S_{2}$ and the reference plane of lens antenna is $S_{3}$. The points of the ray on $S_{1}, S_{2}, S_{3}$ are defined as $P_{1}\left(x_{1}, y_{1}, z_{1}\right), P_{2}\left(x_{2}, y_{2}, z_{2}\right), P_{3}\left(x_{3}, y_{3}, z_{3}\right)$, respectively.

Snell's law is expressed as follows:

$$
k_{1} \sin \theta_{1}=k_{2} \sin \theta_{2}
$$

where $k_{1}$ and $k_{2}$ are the propagation constants within the outside and inside region of lens, and $\theta_{1}$ and $\theta_{2}$ are the incident and the refractive angles, respectively. Applying Eq. (1) to the ray tracing method, the points $P_{1}, P_{2}$ and $P_{3}$ on $S_{1}, S_{2}$, and $\mathrm{S}_{3}$ can be obtained straightforwardly [2].

Fresnel's transmission coefficients of parallel and perpendicular polarization are given by the following expressions, respectively [3].

$$
\begin{gathered}
T_{T M}=\frac{2 \mu_{2} n \cos \theta_{1}}{\mu_{1} n^{2} \cos \theta_{1}+\mu_{2} \sqrt{n^{2}-\sin ^{2} \theta_{1}}} \\
T_{T E}=\frac{2 \mu_{2} \cos \theta_{1}}{\mu_{2} \cos \theta_{1}+\mu_{1} \sqrt{n^{2}-\sin ^{2} \theta_{1}}}
\end{gathered}
$$

where $n$ is the refractive index of lens. 


$$
n=\frac{k_{2}}{k_{1}}=\sqrt{\frac{\mu_{2}}{\mu_{1}}} \sqrt{\frac{\varepsilon_{2}-j\left(\sigma_{2} / \omega\right)}{\varepsilon_{1}-j\left(\sigma_{1} / \omega\right)}}
$$

By using Eqs. (1) - (4), the incident electric field on $P_{1}$ is expressed as follows.

$$
\begin{gathered}
\boldsymbol{E}_{P_{1}}^{\text {in }}=\left(E_{P_{1}}^{x} \boldsymbol{i}_{x}+E_{P_{1}}^{y} \boldsymbol{i}_{y}+E_{P_{1}}^{z} \boldsymbol{i}_{z}\right) \psi \\
E_{P_{1}}^{x}\left(x_{1}, y_{1}\right)=\frac{k_{1}^{2}}{4 \pi R_{1}^{2}}\left(x_{1}-x_{0}\right)\left(y_{1}-y_{0}\right) J_{y}\left(x_{0}, y_{0}\right) \\
E_{P_{1}}^{y}\left(x_{1}, y_{1}\right)=\frac{1}{4 \pi}\left[\left\{\frac{k_{1}^{2}}{R_{1}^{2}}\left(y_{1}-y_{0}\right)^{2}-j \omega \mu_{0}\right\} J_{y}\left(x_{0}, y_{0}\right)+\frac{j k_{1}}{R_{1}}\left(z_{1}-z_{0}\right) M_{x}\left(x_{0}, y_{0}\right)\right] \\
E_{P_{1}}^{z}\left(x_{1}, y_{1}\right)=\frac{1}{4 \pi}\left\{\frac{k_{1}^{2}}{R_{1}^{2}}\left(y_{1}-y_{0}\right)\left(z_{1}-z_{0}\right) J_{y}\left(x_{0}, y_{0}\right)-\frac{j k_{1}}{R_{1}}\left(y_{1}-y_{0}\right) M_{x}\left(x_{0}, y_{0}\right)\right\} \\
\psi=\frac{\exp \left(-j k R_{1}\right)}{R_{1}}
\end{gathered}
$$

The electric field on the reference plane $S_{3}$ is obtained as,

$$
\begin{gathered}
\boldsymbol{E}_{P_{3}}=\left(E_{P_{3}}^{x} \boldsymbol{i}_{x}+E_{P_{3}}^{y} \boldsymbol{i}_{y}+E_{P_{3}}^{z} \boldsymbol{i}_{z}\right) \psi \\
E_{P_{3}}^{i}\left(x_{3}, y_{3}\right)=\left\{K_{x}^{i} E_{P_{1}}^{x}\left(x_{1}, y_{1}\right)+K_{y}^{i} E_{P_{1}}^{y}\left(x_{1}, y_{1}\right)+K_{z}^{i} E_{P_{1}}^{z}\left(x_{1}, y_{1}\right)\right\} \\
\times \frac{\exp \left\{-j\left(k_{1} R_{1}+k_{2} R_{2}+k_{1} R_{3}\right)\right\}}{R_{1} R_{2} R_{3}}, \quad i=x, y, \text { or } z \\
R_{1}=\overline{P^{\prime} P_{1}}, \quad R_{2}=\overline{P_{1} P_{2}}, \quad R_{3}=\overline{P_{2} P_{3}}
\end{gathered}
$$

$K_{x}^{i}, K_{y}^{i}, K_{z}^{i}$ denote the variables defined by the normal vectors and the transmission coefficients at the points $P_{1}$ and $P_{2}$. The transmission coefficient at each point depends on the mode of incident ray.

The radiated field is calculated by the surface integral on the reference plane $S_{3}$. Replacing the coordinate origin to the point of intersection of $S_{3}$ and the z-axis, the electric field is expressed by

$$
\begin{array}{r}
\boldsymbol{E}(r, \theta, \phi)=\frac{1}{4 \pi} \int_{S_{3}}\left\{-j \omega \mu \psi\left(\boldsymbol{n}^{\prime} \times \boldsymbol{H}\right)+\left(\boldsymbol{n}^{\prime} \times \boldsymbol{E}\right) \times \nabla^{\prime} \psi+\left(\boldsymbol{n}^{\prime} \cdot \boldsymbol{E}\right) \nabla^{\prime} \psi\right\} d S^{\prime} \\
=\frac{\exp (-j k r)}{r} \boldsymbol{D}(\theta, \phi) \\
\boldsymbol{D}(\theta, \phi)=\frac{j k_{1}}{4 \pi} \int_{S_{3}}\left[\left\{E_{P_{3}}^{x}\left(x_{3}, y_{3}\right) \cos \phi+E_{P_{3}}^{y}\left(x_{3}, y_{3}\right) \sin \phi\right\} \boldsymbol{i}_{\theta}-\left\{E_{P_{3}}^{x}\left(x_{3}, y_{3}\right) \cos \theta \sin \phi\right.\right. \\
\left.\left.-E_{P_{3}}^{y}\left(x_{3}, y_{3}\right) \cos \theta \cos \phi\right\} \boldsymbol{i}_{\phi}\right] \exp \left\{j k_{1}\left(x_{3} \sin \theta \cos \phi+y_{3} \sin \theta \sin \phi\right)\right\} d S^{\prime}
\end{array}
$$

\section{Numerical and experimental results}

Figure 2 shows an example of dielectric lens antenna and its primary radiator. The lens is made of ABS resin. The diameter, the central thickness and the focal distance of lens are $2 h=60 \mathrm{~mm}, T=20 \mathrm{~mm}$, and $F=45 \mathrm{~mm}$, respectively. The inner surface is flat and the outer convex surface is rotationally symmetric with respect to the z-axis. The relative permittivity of $\mathrm{ABS}$ resin is 2.641 at the frequency of $76.5 \mathrm{GHz}$. The 
geometry of lens antenna is designed by using the optical design software "CODE V" [4]. The primary radiator is the conical horn antenna fed by the rectangular waveguide. The aperture plane of conical horn is located on the $z=z_{0}$ plane. The size of waveguide is $2.54 \times 1.27 \mathrm{~mm}$. The diameter of the aperture of conical horn is $6 \mathrm{~mm}$. The conical horn antenna is excited by the dominant $\mathrm{TE}_{10}$ mode of rectangular waveguide [5].

Figure 3 shows the electric field radiation patterns of lens antenna. The distance between the lens and the aperture of horn is equal to the focal distance of lens. Figure 4 shows the calculated electric field radiation patterns for different $z_{1}-z_{0}$.

\section{Conclusion}

The ray tracing method has proposed to analyze the arbitrarily shaped dielectric lens antenna. Although the reflection loss is considered in the calculation, the multiple reflections within the lens are not considered. As an example of numerical calculation, the radiation patterns of the dielectric lens antenna fed by the conical horn antenna have been shown.

\section{References}

[1] K. Nishikawa: "Antennas and propagation for ITS (Intelligent Transport System)", Proc. of Communication Society Conf. of IEICE, TB-1-6, Oct. 1998 (in Japanese).

[2] T. Kusakawa: "Lens optics Theory and practical program", Tokai University Publishing, pp.1-2, 85-86, 98-100, 1988 (in Japanese).

[3] Institute of Electronics and Communication Engineers of Japan: "Antenna engineering handbook", pp.11-12, 1980 (in Japanese).

[4] http://www.opticalres.com/

[5] C. Balanis: "Antenna theory Analysis and design", Chapter 12, John Wiley \& Sons, 1982.

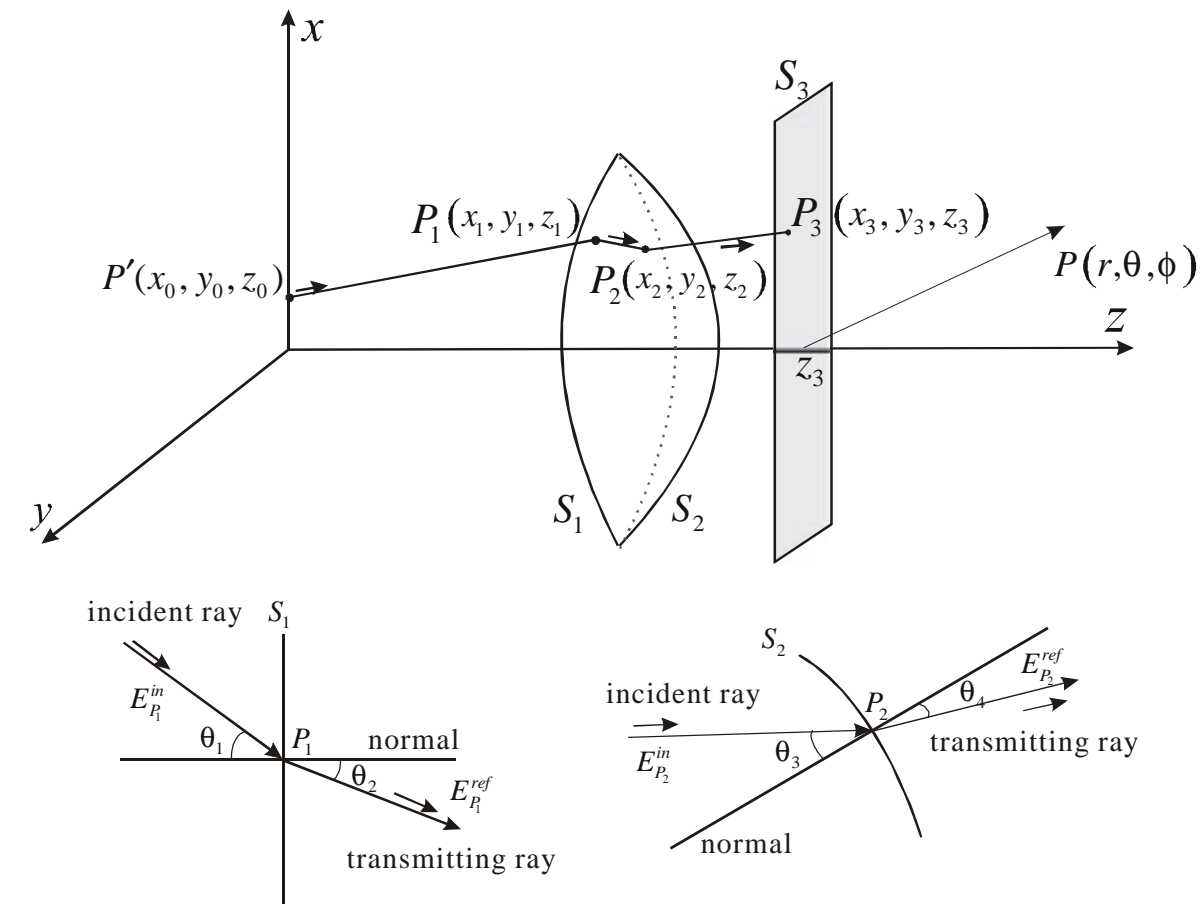

Figure 1. Coordinate system for ray tracing method. 


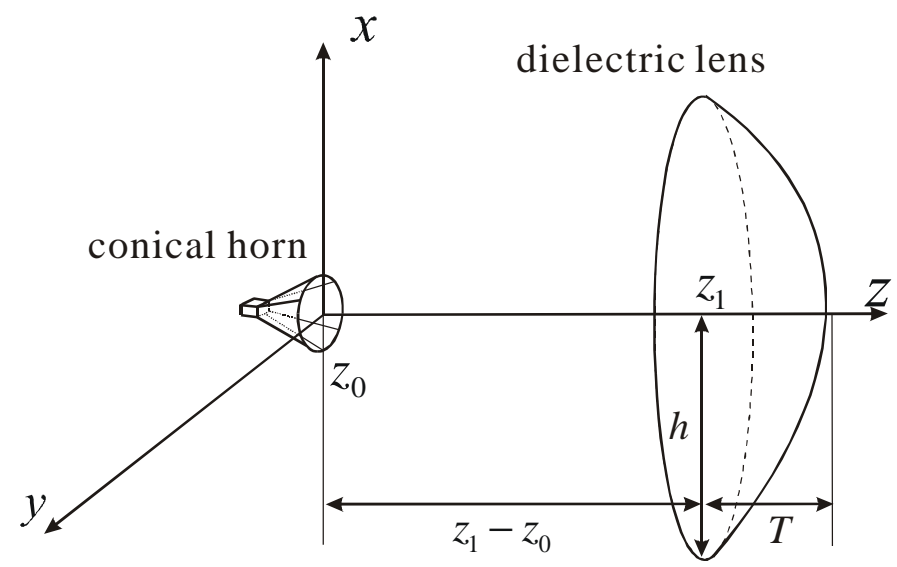

Figure 2. Dielectric lens antenna.

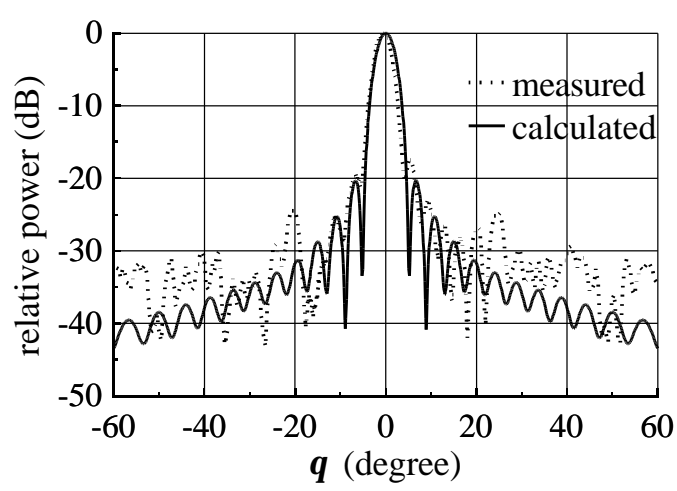

(a) E-plane patterns

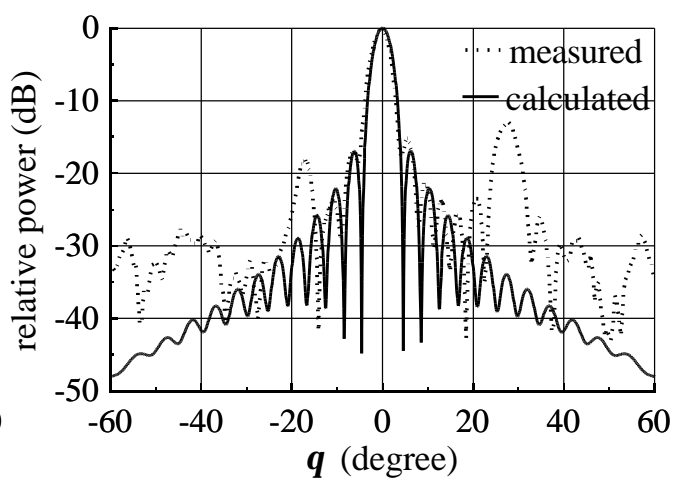

(b) H-plane patterns

Figure 3. Electric field radiation patterns of dielectric lens antenna. ( $2 h=60 \mathrm{~mm}, T=20 \mathrm{~mm}, F=45 \mathrm{~mm}, z_{1}-z_{0}=45 \mathrm{~mm}$ )

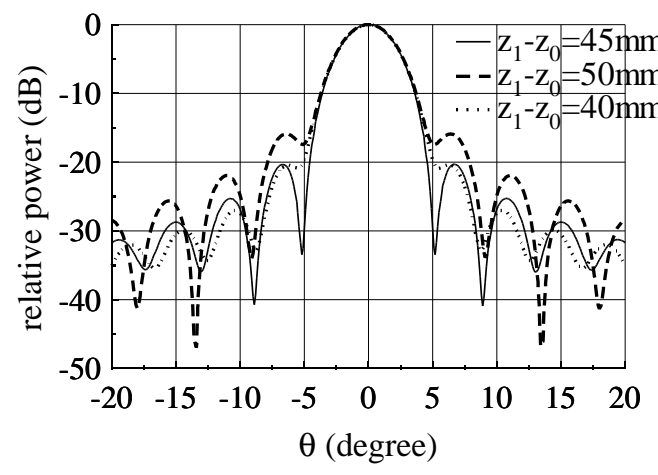

(a) E-plane patterns

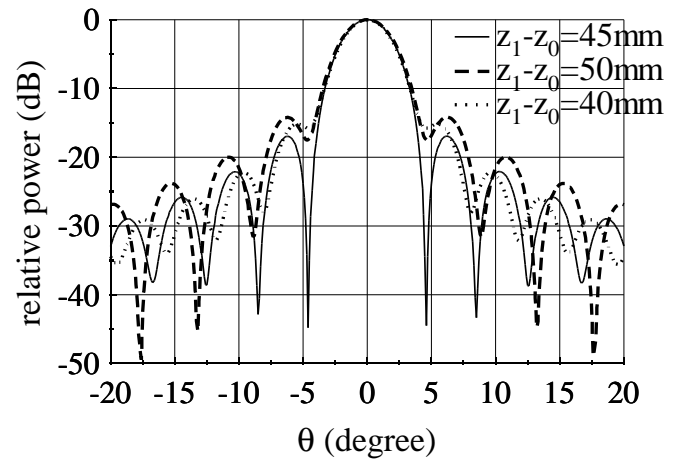

(b) H-plane patterns

Figure 4. Electric field radiation patterns of dielectric lens antenna. ( $2 h=60 \mathrm{~mm}, T=20 \mathrm{~mm}, F=45 \mathrm{~mm})$ 